\title{
Modelling of uncertainties in ultrasound sensor locations in photoacoustic tomography
}

Sahlström, Teemu, Pulkkinen, Aki, Tick, Jenni, Leskinen, Jarkko, Tarvainen, Tanja

Teemu Sahlström, Aki Pulkkinen, Jenni Tick, Jarkko Leskinen, Tanja Tarvainen, "Modelling of uncertainties in ultrasound sensor locations in photoacoustic tomography," Proc. SPIE 11240, Photons Plus Ultrasound: Imaging and Sensing 2020, 112402L (17 February 2020); doi: $10.1117 / 12.2543024$

SPIE. Event: SPIE BiOS, 2020, San Francisco, California, United States 


\title{
Modelling of uncertainties in ultrasound sensor locations in photoacoustic tomography
}

\author{
Teemu Sahlström ${ }^{\mathrm{a}}$, Aki Pulkkinen ${ }^{\mathrm{a}}$, Jenni Tick $^{\mathrm{a}}$, Jarkko Leskinen ${ }^{\mathrm{a}}$, and Tanja Tarvainen ${ }^{\mathrm{a}, \mathrm{b}}$ \\ a Department of Applied Physics, University of Eastern Finland, P.O. Box 1627, 70211 Kuopio, \\ Finland \\ ${ }^{\mathrm{b}}$ Department of Computer Science, University College London, Gower Street, London WC1E \\ 6BT, United Kingdom
}

\begin{abstract}
Photoacoustic tomography (PAT) is an imaging modality developed during the past few decades. In the inverse problem of PAT, the aim is to estimate the spatial distribution of an initial pressure $p_{0}$ generated by the photoacoustic effect, when photoacoustic time-series $p_{t}$ measured on the boundary of the imaged target are given. To produce accurate photoacoustic images, the forward model linking $p_{0}$ to $p_{t}$ has to model the measurement setup and the underlying physics to a sufficient accuracy. Use of an inaccurate model can lead to significant errors in the solution of the inverse problem. In this work, we study the effect and compensation of modelling errors due to uncertainties in ultrasound sensor locations in PAT using Bayesian approximation error modelling. The approach is evaluated with simulated and experimental data using various levels of measurement noise, uncertainties in sensor locations and varying sensor geometries. The results indicate that even small errors in the modelling of ultrasound sensor locations can lead to large errors in the solution of the inverse problem. Furthermore, the magnitude of these errors is affected by the amount of measurement noise and the measurement geometry. The modelling errors can, however, be well compensated by the approximation error modelling.
\end{abstract}

Keywords: Photoacoustic tomography, inverse problems, Bayesian methods, error modelling

\section{INTRODUCTION}

Photoacoustic tomography (PAT) is an imaging modality that utilises the photoacoustic effect. In PAT, the imaged target is illuminated with a short light pulse. Absorption of this light creates areas of local thermal expansion resulting in a localised pressure distribution within the target. This pressure relaxes as ultrasonic waves which are measured on the boundary of the imaged target. In the inverse problem of PAT, the measured ultrasound waves $p_{t}$ are used to estimate the initial pressure distribution $p_{0}$ within the target volume. Applications of PAT include, for example, imaging of vasculature, cancer detection and small animal imaging in biomedical applications. ${ }^{1-3}$

To produce accurate images in PAT, the measurement setup and the underlying physics have to be modelled to a sufficient accuracy. This, however, is not always possible since some model parameters may not be known exactly or the numerical solution of the model is computationally too expensive. Use of an inaccurate forward model can lead to significant errors in the solution of the inverse problem. Modelling errors in PAT have previously been studied in cases such as a variable speed of sound ${ }^{4-6}$ and a finite ultrasound transducer size..$^{7,8}$ In this work, the effect and compensation of modelling errors due to uncertainties in ultrasound sensor locations are studied. The inverse problem of PAT is approached in a Bayesian framework. Compensation of the modelling errors is carried out using Bayesian approximation error modelling. ${ }^{9,10}$

The rest of the paper is structured as follows. The forward model for PAT is described in Section 2. The Bayesian approach to the inverse problem of PAT and the Bayesian approximation error modelling are described in Section 3. Furthermore, results of the simulation and experimental studies are presented in Sections 4 and 5. Finally, the results are concluded in Section 6.

Photons Plus Ultrasound: Imaging and Sensing 2020, edited by Alexander A. Oraevsky, Lihong V. Wang, Proc. of SPIE Vol. 11240, 112402L · C 2020 SPIE · CCC code: 1605-7422/20/\$21 · doi: 10.1117/12.2543024 


\section{FORWARD MODEL}

Propagation of ultrasound waves generated by an initial pressure $p_{0}$ in a homogeneous non-attenuating medium is described by an initial value problem ${ }^{11}$

$$
\left\{\begin{array}{l}
\nabla^{2} p(r, t)-\frac{1}{c^{2}} \frac{\partial^{2} p(r, t)}{\partial t^{2}}=0 \\
p(r, t=0)=p_{0}(r) \\
\frac{\partial}{\partial t} p(r, t=0)=0
\end{array}\right.
$$

where $p$ is the acoustic pressure, $r$ is the location, $t$ denotes the time and $c$ is the speed of sound. In this work, the initial value problem (1) is solved using a pseudospectral $k$-space time-domain method implemented in the k-Wave toolbox. ${ }^{12}$

\section{INVERSE PROBLEM}

In the inverse problem of PAT, the aim is to estimate the initial pressure $p_{0} \in \mathbb{R}^{m}$, when the measured photoacoustic time-series $p_{t} \in \mathbb{R}^{n}$ are given. The discrete observation model for PAT, which links $p_{0}$ to $p_{t}$, can be written as

$$
p_{t}=K p_{0}+e,
$$

where $K \in \mathbb{R}^{n \times m}$ is a discrete forward operator and $e \in \mathbb{R}^{n}$ is additive measurement noise. In this work, the forward operator $K$ is constructed by simulating impulse responses from each of the pixels in the discretised reconstruction domain and placing the resulting wave-forms on the columns of the forward operator.

In this work, the inverse problem in PAT is approached in the Bayesian framework. According to the Bayes' theorem, the solution of the inverse problem, i.e. the posterior distribution, can be written as

$$
\pi\left(p_{0} \mid p_{t}\right)=\frac{\pi\left(p_{t} \mid p_{0}\right) \pi\left(p_{0}\right)}{\pi\left(p_{t}\right)} \propto \pi\left(p_{t} \mid p_{0}\right) \pi\left(p_{0}\right)
$$

where $\pi\left(p_{t} \mid p_{0}\right)$ is the likelihood distribution, $\pi\left(p_{0}\right)$ is the prior distribution and $\pi\left(p_{t}\right)$ is a normalisation constant. Let us now assume that the measurement noise $e$ and the initial pressure $p_{0}$ are mutually independent. Let us further model the measurement noise $e$ as Gaussian distributed $e \sim \mathcal{N}\left(\eta_{e}, \Gamma_{e}\right)$ where $\eta_{e}$ and $\Gamma_{e}$ are the mean and covariance of the measurement noise. Then, the likelihood density can be written $\operatorname{as}^{9}$

$$
\pi\left(p_{t} \mid p_{0}\right) \propto \exp \left\{-\frac{1}{2}\left\|L_{e}\left(p_{t}-K p_{0}-\eta_{e}\right)\right\|_{2}^{2}\right\}
$$

where $L_{e}$ is the Cholesky decomposition of the inverse covariance matrix of the measurement noise $\Gamma_{e}^{-1}=L_{e}^{\mathrm{T}} L_{e}$. In this work, the noise model (4) is referred to as the conventional error model (CEM). Let us now model the prior density as Gaussian distributed $p_{0} \sim \mathcal{N}\left(\eta_{p_{0}}, \Gamma_{p_{0}}\right)$ where $\eta_{p_{0}}$ and $\Gamma_{p_{0}}$ are the mean and covariance of the prior distribution. In this case, the posterior density, given by (3), can be written as ${ }^{9}$

$$
\pi\left(p_{0} \mid p_{t}\right) \propto \exp \left\{-\frac{1}{2}\left(\left\|L_{e}\left(p_{t}-K p_{0}-\eta_{e}\right)\right\|_{2}^{2}+\left\|L_{p_{0}}\left(p_{0}-\eta_{p_{0}}\right)\right\|_{2}^{2}\right)\right\},
$$

where $L_{p_{0}}$ is the Cholesky decomposition of the inverse covariance matrix of the prior distribution $\Gamma_{p_{0}}^{-1}=L_{p_{0}}^{\mathrm{T}} L_{p_{0}}$. The posterior density (5) is a Gaussian distribution ${ }^{9}$

$$
p_{0} \mid p_{t} \sim \mathcal{N}\left(\eta_{p_{0} \mid p_{t}}, \Gamma_{p_{0} \mid p_{t}}\right)
$$

where

$$
\begin{aligned}
\eta_{p_{0} \mid p_{t}} & =\Gamma_{p_{0} \mid p_{t}}\left(K^{\mathrm{T}} \Gamma_{e}^{-1}\left(p_{t}-\eta_{e}\right)+\Gamma_{p_{0}}^{-1} \eta_{p_{0}}\right) \\
\Gamma_{p_{0} \mid p_{t}} & =\left(\Gamma_{p_{0}}^{-1}+K^{\mathrm{T}} \Gamma_{e}^{-1} K\right)^{-1}
\end{aligned}
$$




\subsection{Bayesian Approximation Error Approach}

Ideally, the forward operator $K$ should be constructed such that it takes into account all underlying physics and parameters of the measurement setup. In practice, however, it can contain modelling errors arising from, for example, coarse numerical discretisation, simplifications of the numerical model or uncertainties in the model parameters.

Let us now consider a situation, where the forward operator $K$ is parameterised by some, possibly inaccurately known, parameter $\gamma$, i.e. $K \rightarrow K(\gamma)$, and the observation model (2) is written as

$$
p_{t}=K(\gamma) p_{0}+e .
$$

In practice, the parameter $\gamma$ is often fixed to some constant $\gamma \rightarrow \gamma_{0}$ and a forward model $K\left(\gamma_{0}\right)$ is used instead. In this case, the observation model is written as

$$
p_{t}=K\left(\gamma_{0}\right) p_{0}+e .
$$

If the fixed parameter $\gamma_{0}$ is not accurate, use of this observation model can result in errors in the solution of the inverse problem, especially in the case of an ill-posed problem. Possible uncertainties in $\gamma$ can, however, be taken in to account by using the Bayesian approximation error modelling, in which information about the uncertainties is incorporated to the likelihood model. In the approximation error approach, the observation model (9) is written as

$$
\begin{aligned}
p_{t} & =K\left(\gamma_{0}\right) p_{0}+\left(K(\gamma)-K\left(\gamma_{0}\right)\right) p_{0}+e \\
& =K\left(\gamma_{0}\right) p_{0}+\varepsilon+e \\
& =K\left(\gamma_{0}\right) p_{0}+n,
\end{aligned}
$$

where $\varepsilon$ is the approximation error describing the discrepancy between the accurate and inaccurate models and $n=\varepsilon+e$ is the total error.

Let us now ignore the mutual dependence of the approximation error $\varepsilon$ and the initial pressure $p_{0}$. Let us further model the approximation error $\varepsilon$ as Gaussian distributed $\varepsilon \sim \mathcal{N}\left(\eta_{\varepsilon}, \Gamma_{\varepsilon}\right)$ where $\eta_{\varepsilon}$ is the mean and $\Gamma_{\varepsilon}$ is the covariance of the approximation error. Then, the total error $n$ is also Gaussian distributed $n \sim \mathcal{N}\left(\eta_{n}, \Gamma_{n}\right)$ where $\eta_{n}=\eta_{\varepsilon}+\eta_{e}$ is the mean and $\Gamma_{n}=\Gamma_{\varepsilon}+\Gamma_{e}$ is the covariance of the total error. In this case, the likelihood density can be written as ${ }^{9}$

$$
\pi\left(p_{t} \mid p_{0}\right) \propto \exp \left\{-\frac{1}{2}\left\|L_{n}\left(p_{t}-K\left(\gamma_{0}\right) p_{0}-\eta_{n}\right)\right\|_{2}^{2}\right\} .
$$

The noise model (14) is referred to as the enhanced error model (EEM). Let us now model the prior density $\pi\left(p_{0}\right)$ as Gaussian distributed. The posterior distribution (3) can then be written as ${ }^{9}$

$$
\pi\left(p_{0} \mid p_{t}\right) \propto \exp \left\{-\frac{1}{2}\left(\left\|L_{n}\left(p_{t}-K\left(\gamma_{0}\right) p_{0}-\eta_{n}\right)\right\|_{2}^{2}+\left\|L_{p_{0}}\left(p_{0}-\eta_{p_{0}}\right)\right\|_{2}^{2}\right)\right\}
$$

where $L_{n}$ is the Cholesky decomposition of the inverse covariance matrix of the total error $\Gamma_{n}^{-1}=L_{n}^{\mathrm{T}} L_{n}$. This is a Gaussian distribution

$$
p_{0} \mid p_{t} \sim \mathcal{N}\left(\eta_{p_{0} \mid p_{t}}, \Gamma_{p_{0} \mid p_{t}}\right)
$$

where

$$
\begin{aligned}
\eta_{p_{0} \mid p_{t}} & =\Gamma_{p_{0} \mid p_{t}}\left(K\left(\gamma_{0}\right)^{\mathrm{T}} \Gamma_{n}^{-1}\left(p_{t}-\eta_{n}\right)+\Gamma_{p_{0}}^{-1} \eta_{p_{0}}\right) \\
\Gamma_{p_{0} \mid p_{t}} & =\left(\Gamma_{p_{0}}^{-1}+K\left(\gamma_{0}\right)^{\mathrm{T}} \Gamma_{n}^{-1} K\left(\gamma_{0}\right)\right)^{-1} .
\end{aligned}
$$

Statistics of the approximation error can be computed, for example, as a sample based estimation as follows. Let $M=\left\{m^{(1)}, m^{(2)}, \cdots, m^{(L)}\right\}$ be a set of samples drawn from the prior distribution $\pi\left(p_{0}\right)$. Furthermore, let 


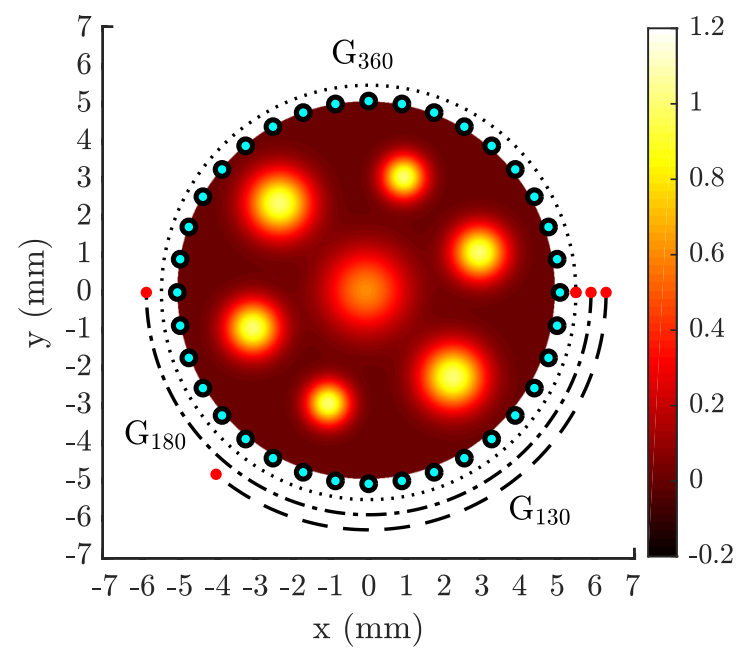

(a)

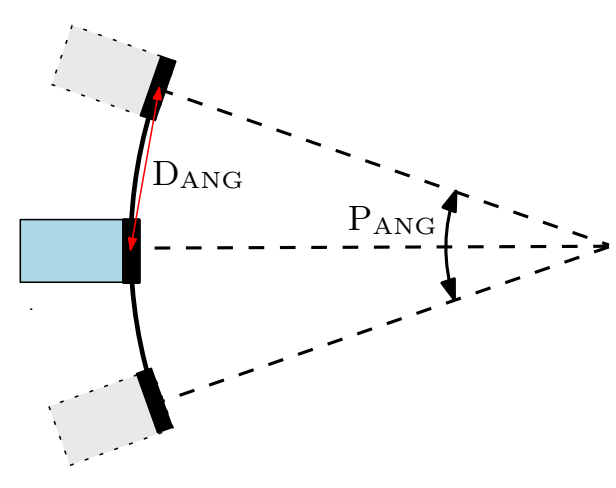

(b)

Figure 1: (a) Simulated initial pressure and sensor geometries. The sensor points are indicated as light blue points on the boundary of the circular domain. Full-view geometry $\left(\mathrm{G}_{360}\right)$ and the limited view geometries $\left(\mathrm{G}_{180}\right.$ and $\left.\mathrm{G}_{130}\right)$ are indicated with arc spanning $360^{\circ}$ (dotted line), $180^{\circ}$ (dash-dotted line) and $130^{\circ}$ (dashed line) of the target domain respectively. (b) Visualisation of the angularly altered sensor locations $\mathrm{P}_{\mathrm{ANG}}$. The unaltered sensor location is shown as the light blue rectangle with solid outlines and the altered sensor locations are shown with gray rectangles with dotted outlines. The distance between the unaltered and altered sensor locations is indicated with the red line.

$\gamma^{(l)}, l=1, \ldots, L$ be a sampled value of the uncertain parameter $\gamma$. Estimates for the mean and covariance of the approximation error $\varepsilon$ can then be computed as ${ }^{9}$

$$
\begin{aligned}
& \eta_{\varepsilon}=\frac{1}{N} \sum_{l=1}^{L} \varepsilon^{(l)} \\
& \Gamma_{\varepsilon}=\frac{1}{N-1} \sum_{l=1}^{L} \varepsilon^{(l)}\left(\varepsilon^{(l)}\right)^{\mathrm{T}}-\eta_{\varepsilon} \eta_{\varepsilon}^{\mathrm{T}},
\end{aligned}
$$

where

$$
\varepsilon^{(l)}=K\left(\gamma^{(l)}\right) m^{(l)}-K\left(\gamma_{0}\right) m^{(l)} .
$$

\section{SIMULATIONS}

In the simulation study, the effect and compensation of modelling errors due to uncertainties in ultrasound sensor locations were studied using three sensor geometries, three levels of uncertainties and multiple levels of measurement noise.

\subsection{Data Simulation}

In the simulations, a $10 \mathrm{~mm}$ diameter circular domain was considered. The simulated initial pressure $p_{0}$, visualised in Fig. 1a, consisted of seven smooth inclusion with peak amplitudes of 1 (outermost inclusions) and 0.6 (middle inclusion). The speed of sound was set to $c=1500 \mathrm{~m} / \mathrm{s}$.

The sensor geometries used in the simulations consisted of 36, 19 and 14 ideal point-like ultrasound sensors distributed on $360^{\circ}, 180^{\circ}$ and $130^{\circ}$ arcs $\left(10^{\circ}\right.$ separation $)$ around the boundary of the domain. These sensor geometries are referred to as $\mathrm{G}_{360}, \mathrm{G}_{180}$ and $\mathrm{G}_{130}$ respectively. Illustration of the sensor geometries is shown in Fig. 1a. The ultrasound sensor locations were defined using cartesian coordinates. In the situations, where the sensor locations did not match the spatial discretisation, the recorded waveforms were interpolated using a piecewise 
Table 1: Uncertainties $\mathrm{P}_{\mathrm{ANG}, 1}-\mathrm{P}_{\mathrm{ANG}, 3}$, the corresponding uniform distribution used in alteration of the sensor positions and the distance of the maximum alteration from the unaltered sensor position $\mathrm{D}_{\mathrm{ANG}}$.

\begin{tabular}{ccc}
\hline Uncertainty & \multicolumn{1}{c}{ Distribution } & $\mathrm{D}_{\text {ANG }}(\mu \mathrm{m})$ \\
\hline \hline $\mathrm{P}_{\mathrm{ANG}, 1}$ & $\operatorname{Unif}\left(\left[-1^{\circ},-0.5^{\circ}\right] \cup\left[0.5^{\circ}, 1^{\circ}\right]\right)$ & 89 \\
$\mathrm{P}_{\mathrm{ANG}, 2}$ & $\operatorname{Unif}\left(\left[-2^{\circ},-1^{\circ}\right] \cup\left[1^{\circ}, 2^{\circ}\right]\right)$ & 177 \\
$\mathrm{P}_{\mathrm{ANG}, 3}$ & $\operatorname{Unif}\left(\left[-3^{\circ},-1.5^{\circ}\right] \cup\left[1.5^{\circ}, 3^{\circ}\right]\right)$ & 266 \\
\hline
\end{tabular}

Table 2: Noise levels $\sigma_{e}$ used in the simulation study: percentages of the maximum amplitudes E$_{\%}$ and the corresponding signal-to-noise ratios (SNR).

\begin{tabular}{lcccccccccc}
\hline \multicolumn{11}{c}{$\sigma_{e}$} \\
\hline \hline E $_{\%}(\%)$ & 0.5 & 1 & 1.5 & 2 & 2.5 & 3 & 3.5 & 4 & 4.5 & 5 \\
SNR $(\mathrm{dB})$ & 34.9 & 28.9 & 25.4 & 22.9 & 21.0 & 19.4 & 18.1 & 16.9 & 15.9 & 14.9 \\
\hline
\end{tabular}

linear triangular discretisation. To simulate uncertainties in ultrasound sensor locations, the photoacoustic data was simulated using angularly altered sensor locations (illustrated in Fig. 1b). The alterations were drawn from three uniform distributions $\mathrm{P}_{\mathrm{ANG}, 1}, \mathrm{P}_{\mathrm{ANG}, 2}$ and $\mathrm{P}_{\mathrm{ANG}, 3}$ shown in Table 1. The altered sensor locations were drawn separately for each sensor.

For the simulation of the photoacoustic data, a square $10.4 \mathrm{~mm} \times 10.4 \mathrm{~mm}$ computation domain was discretised into $416 \times 416$ pixels with a side length of $\Delta x=25 \mu \mathrm{m}$. The discretisation was extended with a 600 $\mu \mathrm{m}$ thick (24 pixels) perfectly matched layer (PML) to absorb reflections on the boundary of the computational domain. The time discretisation was chosen based on a Courant-Friedrichs-Lewy (CFL) number of 0.3, which corresponded to a time step of $5 \mathrm{~ns}$ and a sampling frequency of $200 \mathrm{MHz}$. Number of time points was set to $N_{t}=1652$, which resulted in a flight time of $8260 \mathrm{~ns}$ and a propagation distance of $12.4 \mathrm{~mm}$. Photoacoustic data was simulated by solving the wave-equation (1) using a $k$-space time-domain method implemented in the k-Wave toolbox. ${ }^{12}$

To study the effect of measurement noise $e$, various levels of Gaussian distributed, zero-mean noise was added to the data. In total, ten noise levels $\sigma_{e}$, corresponding to $0.5 \%-5 \%$ with $0.5 \%$ increments of the maximum amplitude of the simulated signal were used. In this work, these noise levels are referred to as $\sigma_{e, 0.5 \%}, \sigma_{e, 1 \%}, \ldots, \sigma_{e, 5 \%}$ respectively. Noise levels and the respective signal-to-noise ratios are given in Table 2.

\subsection{Inverse Problem}

For the inverse problem, a square $10.4 \mathrm{~mm} \times 10.4 \mathrm{~mm}$ computation domain was discretised to $133 \times 133$ pixels with a side length of $\Delta x=78.1 \mu \mathrm{m}$. The discretisation was extended by a $1600 \mu \mathrm{m}$ (21 pixels) thick PML-layer. The time discretisation was chosen based on a CFL number of 0.3. This resulted in a time step of $15.6 \mathrm{~ns}$ and a sampling frequency of $64 \mathrm{MHz}$. Furthermore, the number of time steps was set to $N_{t}=477$ resulting in a flight time of $7453 \mathrm{~ns}$ and a propagation distance of $11.2 \mathrm{~mm}$.

The inverse problem of PAT was solved using (16)-(18) and three different error models as follows. First, the inverse problem was solved using accurately modelled sensor locations, i.e. the same sensor locations that were used to simulate the data ( $\gamma$ is known accurately and $\varepsilon=0$ ). Second, the inverse problem was solved using inaccurately modelled sensor locations assuming the fixed (unaltered) sensor positions without error modelling ( $\gamma=\gamma_{0}$ and $\varepsilon$ assumed to be zero). Third, the inverse problem was solved using inaccurately modelled fixed sensor locations with approximation error modelling $\left(\gamma=\gamma_{0}\right.$ and $\left.\varepsilon \sim \mathcal{N}\left(\eta_{\varepsilon}, \Gamma_{\varepsilon}\right)\right)$. In this work, these three cases are referred to as the conventional error model with accurately modelled sensor locations (ACEM), the 
conventional error model with inaccurately modelled sensor locations (ICEM) and the enhanced error model (EEM), respectively.

The prior model used in the reconstructions was a Gaussian Ornstein-Uhlenbeck process, defined by the covariance function ${ }^{13}$

$$
\Gamma_{p_{0}, i j}=\sigma_{p_{0}}^{2} \exp \left\{-\frac{\left\|r_{i}-r_{j}\right\|}{\ell}\right\}
$$

where $\sigma_{p_{0}}^{2}$ is the variance, $r_{i, j}$ are locations of the pixels and $\ell$ is a characteristic length controlling the length of the spatial correlation. In this work the mean, standard deviation and characteristic length were set as $\eta_{p_{0}}=0.5$, $\sigma_{p_{0}}=0.25$ and $\ell=650 \mu \mathrm{m}$ respectively. In the inverse problem, the measurement noise $e$ was modelled as zero mean with standard deviations $\sigma_{e}$ defined as a percentage of the maximum amplitude of the simulated data.

Statistics for the approximation error $\varepsilon$ were computed as in (19)-(20) using $N=20000$ samples $m^{(l)}$ of the initial pressure $p_{0}$ drawn from the Ornstein-Uhlenbeck prior model. In the situations, where the drawn pixel values were negative, the value was set to zero. Using these samples, the forward solutions corresponding to $K\left(\gamma^{(l)}\right) m^{(l)}$ were computed by drawing sensor positions $\gamma^{(l)}$ from the uniform distributions $\mathrm{P}_{\mathrm{ANG}, 1}-\mathrm{P}_{\mathrm{ANG}, 3}$ and simulating the forward solution using the k-Wave toolbox. Sensor positions were drawn independently for each of the sensors in the sensor geometry. Furthermore, the sensor positions were drawn repeatedly for each of the sample $m^{(l)}$. Forward solutions corresponding to the term $K\left(\gamma_{0}\right) m^{(l)}$ were computed using unaltered sensor locations.

\subsection{Results}

Means of the posterior disributions (reconstructed images) using accurately modelled sensor locations (ACEM), inaccurately modelled sensor locations without error modelling (ICEM) and inaccurately modelled sensor locations with error modelling (EEM), a noise level of $\sigma_{e, 1,5 \%}$, uncertainties $\mathrm{P}_{\mathrm{ANG}, 1}-\mathrm{P}_{\mathrm{ANG}, 3}$ and sensor geometries $\mathrm{G}_{360}$ and $\mathrm{G}_{130}$ are presented in Fig. 2. As it can be seen, the magnitude of the errors and artefacts in the reconstructed images without error modelling (ICEM) are highly dependent on the level of uncertainty. In the case of the lowest uncertainty $\mathrm{P}_{\mathrm{ANG}, 1}$, these errors in the reconstructed images can be observed to be relatively small. However, the errors grow considerably with increasing uncertainty, which can be explained by an increasing magnitude of the modelling errors. In the case of the highest uncertainty $\mathrm{P}_{\mathrm{ANG}, 3}$, the uncertainties result in significant artefacts, that can be seen as distortions spanning the whole image. In addition to the level of uncertainty, the errors in the reconstructed images are affected by the sensor geometry. When comparing the reconstructions obtained using the inaccurately modelled sensor locations (ICEM) with the same level of uncertainty and different sensor geometries, it can be seen that the errors are significantly larger in the case of the limited-view geometry $\mathrm{G}_{130}$ than in the full-view geometry $\mathrm{G}_{360}$. The relatively large errors in the limited-view sensor geometry can be explained by the reduced amount of information and increased ill-posedness of the inverse problem. The errors due to the uncertainties in ultrasound sensor locations are, however, well compensated by the enhanced error model. From the reconstructions computed using approximation error modelling (EEM), it can be seen, that the artefacts are significantly reduced and the locations and amplitudes of the inclusions are corrected close to the accurate reconstruction (ACEM).

Means of the posterior disributions (reconstructed images) using accurately modelled sensor locations (ACEM), inaccurately modelled sensor locations without error modelling (ICEM) and inaccurately modelled sensor locations with error modelling (EEM), with noise levels $\sigma_{e, 0.5 \%}, \sigma_{e, 1.5 \%}$ and $\sigma_{e, 3 \%}$, uncertainty $\mathrm{P}_{\mathrm{ANG}, 3}$ and sensor geometries $\mathrm{G}_{360}$ and $\mathrm{G}_{130}$ are presensted in Fig. 3. As it can be seen, the errors in the reconstructed images are affected by the level of the noise. The errors in the reconstructions using inaccurately modelled sensor locations without error modelling (ICEM) are most significant in the case of the lowest measurement noise of $\sigma_{e, 0.5 \%}$. In this case, significant artefacts in the reconstructions can be seen with both sensor geometries $\mathrm{G}_{360}$ and $\mathrm{G}_{130}$. These errors are, however, reduced in the cases of higher measurement noises $\sigma_{e, 1.5 \%}$ and $\sigma_{e, 3 \%}$. Furthermore, the larger errors due to the limited-view geometry $\mathrm{G}_{130}$, previously observed in Fig. 2, can also be seen. The errors due to uncertainties in ultrasound sensor locations are, however, effectively compensated for every noise level by using the approximation error modelling. 


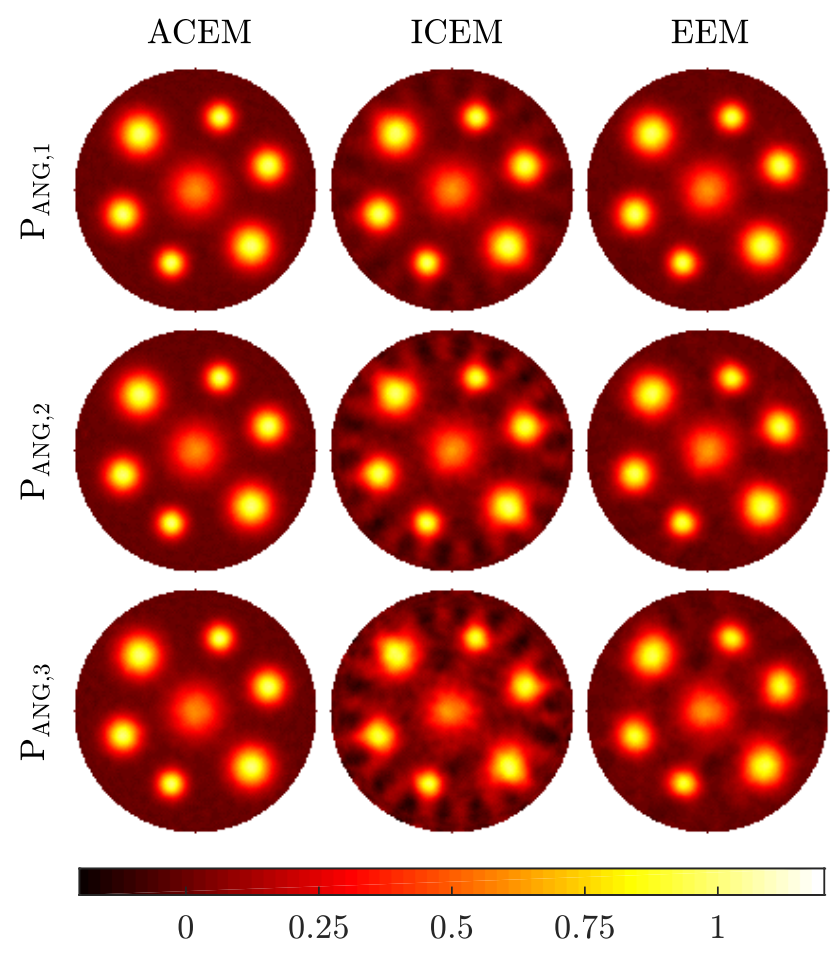

(a) $\mathrm{G}_{360}$

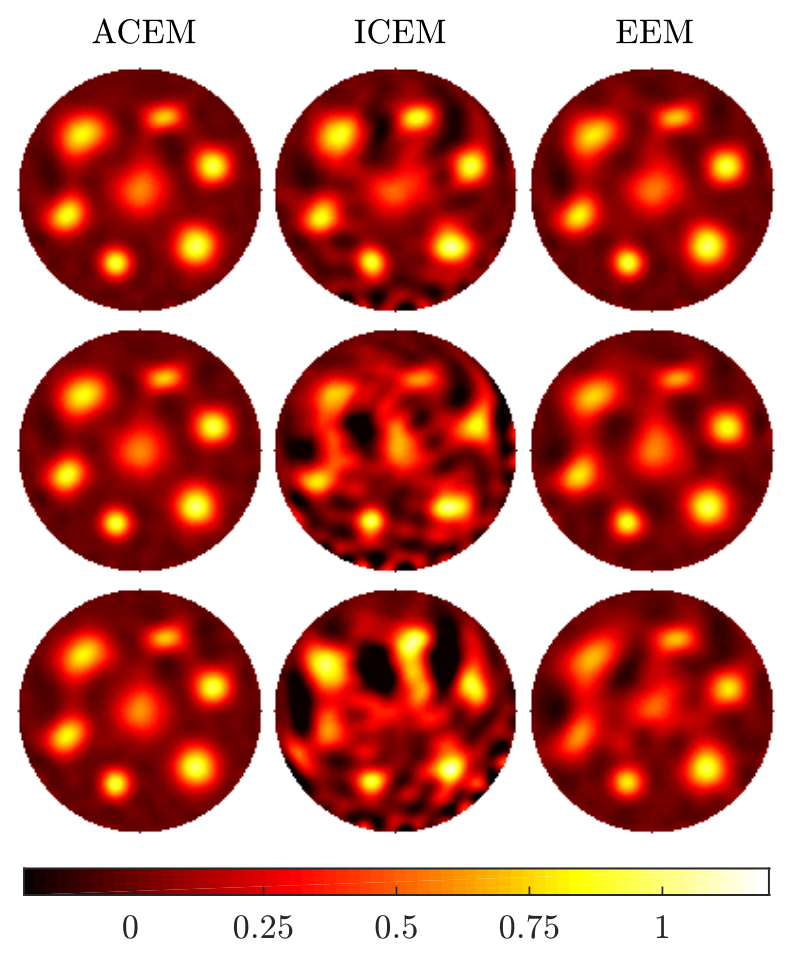

(b) $\mathrm{G}_{130}$

Figure 2: Estimated initial pressure $p_{0}$ using various levels of uncertainties with sensor geometries $\mathrm{G}_{360}(\mathrm{a})$ and $\mathrm{G}_{130}(\mathrm{~b})$ and noise level $\sigma_{e, 1,5 \%}$. For each subfigure from left to right: the conventional error model with accurately modelled sensor locations (ACEM), the conventional error model with inaccurately modelled sensor locations (ICEM) and the enhanced error model (EEM), and from top to bottom, uncertainties $\mathrm{P}_{\mathrm{ANG}, 1}, \mathrm{P}_{\mathrm{ANG}, 2}$ and $\mathrm{P}_{\mathrm{ANG}, 3}$.

Accuracy of the reconstructions was evaluated by computing the relative errors

$$
\mathrm{E}_{p_{0}}=100 \% \cdot \frac{\left\|p_{0, \mathrm{TRUE}}-p_{0, \mathrm{EST}}\right\|}{\left\|p_{0, \mathrm{TRUE}}\right\|}
$$

where $p_{0, \mathrm{TRUE}}$ is the simulated initial pressure and $p_{0, \mathrm{EST}}$ is the estimated initial pressure. These errors, which were computed for all sensor geometries $\mathrm{G}_{360}-\mathrm{G}_{130}$, uncertainties $\mathrm{P}_{\mathrm{ANG}, 1}-\mathrm{P}_{\mathrm{ANG}, 3}$ and levels of measurement noise $\sigma_{e, 0.5 \%}-\sigma_{e, 5 \%}$ are visualised in Fig. 4 .

As it can be seen, the relative errors depend on the level of uncertainty. This is most evident when the modelling errors are ignored. Furthermore, the relative errors are highly dependent on the sensor geometry. The effect of the sensor geometry can be seen as increasing relative errors, as the number of sensors decrease and limited view geometries are used. This effect is most notable in the case, where the inaccurately modelled sensor locations are used and the modelling errors are ignored. It can also be seen that modelling of errors compensates for uncertainties in sensor positions well and the relative errors are smaller than when the uncertainties have been ignored. However, also in these situations, the relative errors increase when uncertainties in sensor positions increase and/or when measurement geometry is limited. It can also be observed, that the relative errors computed using accurately modelled sensor locations (ACEM) and inaccurately modelled sensor locations with error modelling (EEM) increase as a function of increasing measurement noise. On the contrary, the relative errors of the inaccurately modelled sensor locations (ICEM) increase with decreasing noise. This effect can be explained by the ratio of the noise and modelling errors. Depending on the levels of measurement noise and the modelling errors, an inverse problem could be described as either measurement noise or approximation error dominant. ${ }^{9}$ In practice, this means that large noise can cover the effect of modelling errors. On the other hand, if 


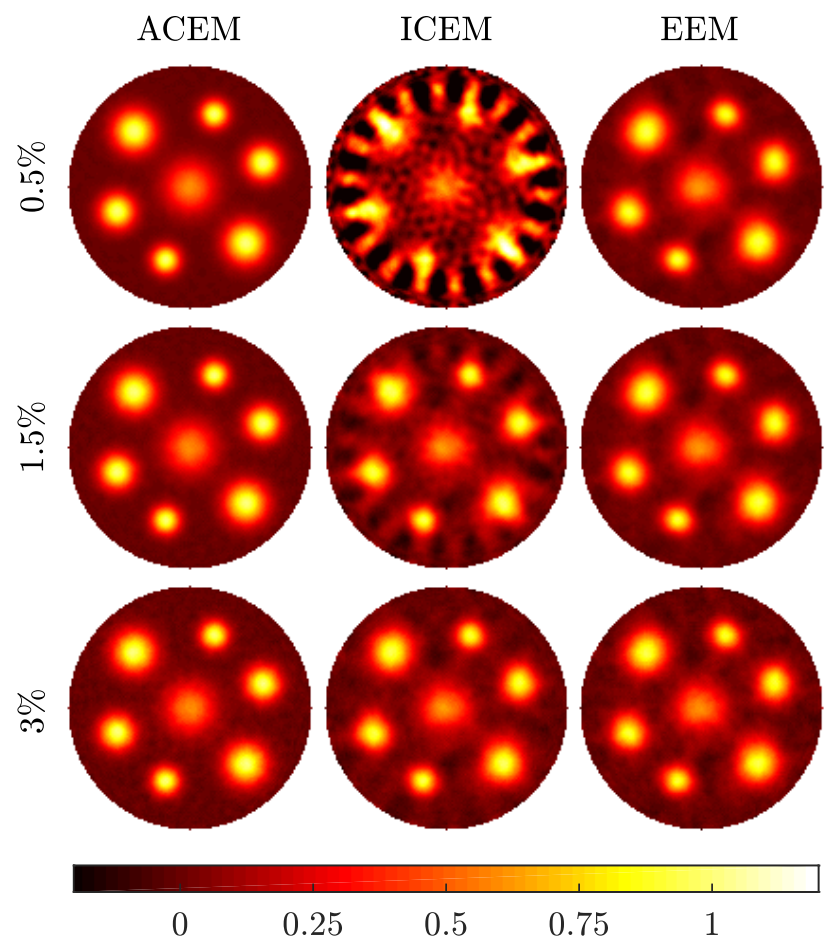

(a) $\mathrm{G}_{360}$

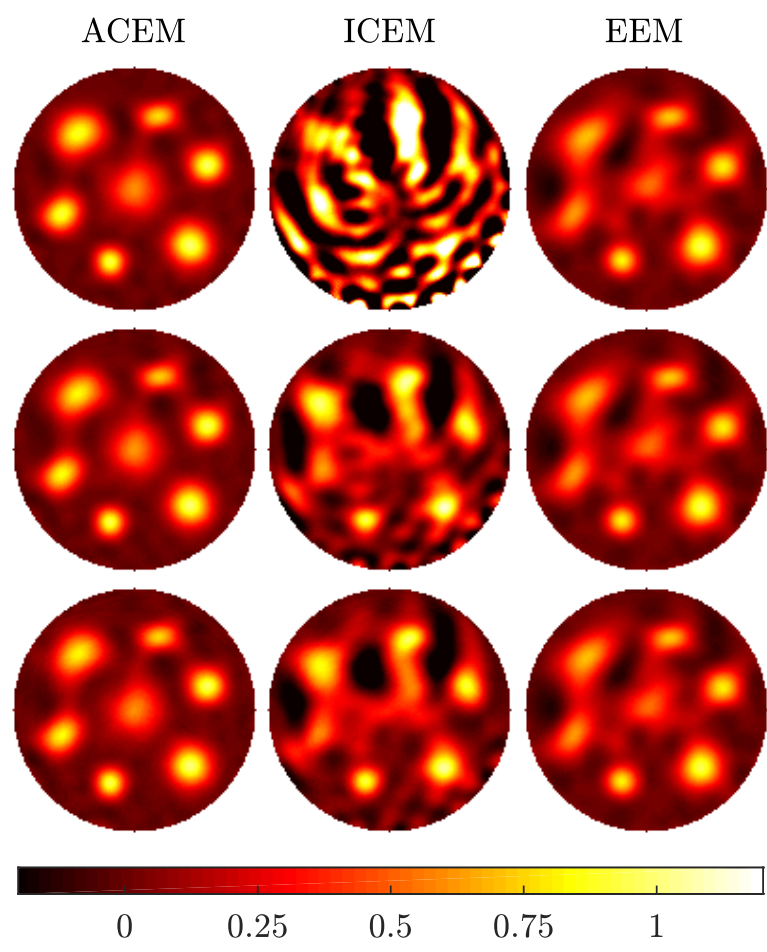

(b) $\mathrm{G}_{130}$

Figure 3: Estimated initial pressure $p_{0}$ using various noise levels with sensor geometries $\mathrm{G}_{360}(\mathrm{a})$ and $\mathrm{G}_{130}$ (b) and uncertainty $\mathrm{P}_{\mathrm{ANG}, 3}$. For each subfigure from left to right: the conventional error model with accurately modelled sensor locations (ACEM), the conventional error model with inaccurately modelled sensor locations (ICEM) and the enhanced error model (EEM), and from top to bottom, noise levels $\sigma_{e, 0.5 \%}, \sigma_{e, 1.5 \%}$ and $\sigma_{e, 3 \%}$.

the approximation errors are significantly larger compared to the measurement noise, ignoring the approximation errors can lead to significant errors in the reconstructed images.

\section{EXPERIMENTAL STUDY}

We used our LED-based photoacoustic tomography system to study the methodology with experimental data. ${ }^{14}$ The LED (model SST-90-R, Luminus Devices, MA, USA) operated at $617 \mathrm{~nm}$ with a pulse energy of $5.26 \pm 0.02 \mu \mathrm{J}$. Photoacoustic waves were recorded using a piezoelectric cylindrically focused ultrasound sensor (model V383, Olympus NDT, MA, USA) with a focal length of $33.0 \mathrm{~mm}$. The imaged target consisted of three parallel plastic microcapillary tubes (polyethylene terephthalate glycol; Globe Scientific, NJ, USA) filled with Indian ink solution (art. no. 44257000, Royal Talens, Apeldoorn, the Netherlands). The ultrasound sensor was rotated in a plane perpendicular to the tubes. Photoacoustic data was recorded on a $180^{\circ} 33.8 \mathrm{~mm}$ diameter arc using $5^{\circ}$ increments. The data was sampled at $50 \mathrm{MHz}$ and was averaged over 1024 consecutive light pulses. The noise level in the data corresponded to $3 \%-22 \%$ of the maximum recorded amplitude depending on the measurement position. The speed of sound was determined according to the temperature of the water and set to $c=1481.7 \mathrm{~m} / \mathrm{s}$.

\subsection{Inverse Problem}

For the inverse problem, a $72.4 \mathrm{~mm} \times 43.3 \mathrm{~mm} \times 10.2 \mathrm{~mm}$ rectangular computation domain was considered. The domain was discretised in $384 \times 230 \times 52$ pixels with a side length of $\Delta x=188.4 \mu \mathrm{m}$. The discretisation was extended with a $1.5 \mathrm{~mm} \times 2.4 \mathrm{~mm} \times 1.9 \mathrm{~mm}$ thick $(8 \times 13 \times 10$ pixels $)$ PML layer. Temporal discretisation was set to match a CFL-number of 0.3 , which resulted in a time step of $38.2 \mathrm{~ns}$. Number of time points was set to $N_{t}=897$, which corresponded to a flight time of $34.3 \mu$ s and a propagation distance of $50.7 \mathrm{~mm}$. 


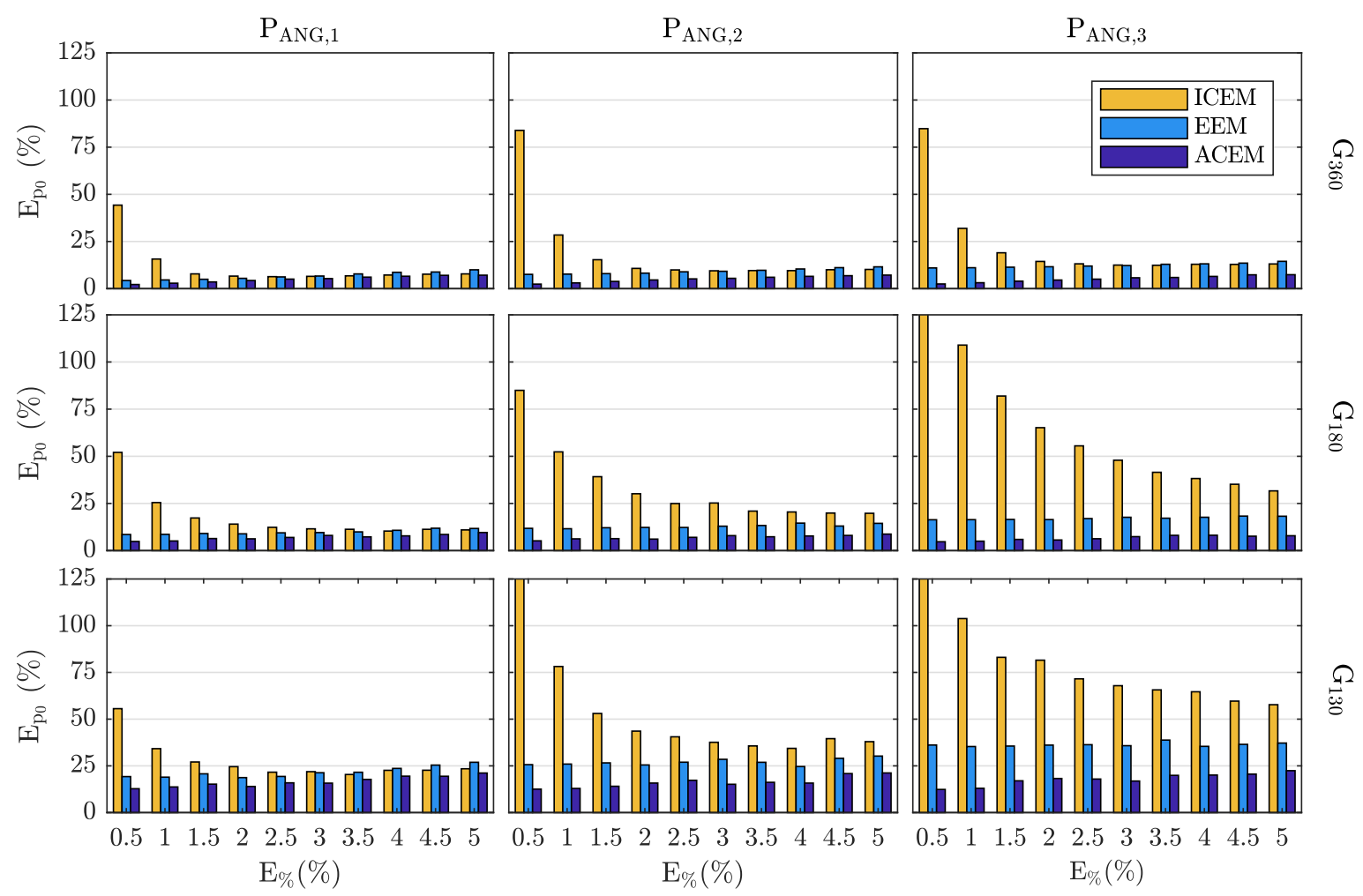

Figure 4: Relative errors $\mathrm{E}_{p_{0}}$ of the estimated initial pressure $p_{0}$ obtained using the accurate conventional error model (ACEM, dark blue bars), the inaccurate conventional error model (ICEM, yellow bars) and the enhanced error model (EEM, light blue bars) and measurement error levels $\mathrm{E}_{\%}$ given in Table 2. From left to right: uncertainties $\mathrm{P}_{\mathrm{ANG}, 1}-$ $\mathrm{P}_{\mathrm{ANG}, 3}$ and from top to bottom sensor geometries $\mathrm{G}_{360}-\mathrm{G}_{130}$.

To simulate uncertainties in the modelling of ultrasound sensor locations, the forward operator $K$ was constructed by altering the radial distance of the ultrasound sensor positions from the central axis of the measurement setup. Alteration for each of the sensors was drawn from a discrete uniform distribution $\mathcal{U}(-188 \mu \mathrm{m}, 0 \mu \mathrm{m}, 188 \mu \mathrm{m})$. The forward operator was constructed by taking advantage of the symmetry of the imaged target. This meant that instead of simulating the impulse responses of individual voxels, lines parallel to the target (z-direction) were used instead. Using this type of approach resulted in a quasi-3D model producing computationally efficient $2 \mathrm{D}$ images while allowing the modelling of the shape of the finite-sized ultrasound sensor. The finite size of the ultrasound sensor was modelled by averaging the recorded waveform over 124 point-sensors arranged to the shape of the ultrasound sensor. The frequency response of the ultrasound sensor was modelled by filtering the signal using the frequency response provided by the manufacturer.

As in the simulation study, the inverse problem was solved using (16)-(18) with accurately modelled sensor locations (ACEM), inaccurately modelled sensor locations without error modelling (ICEM) and inaccurately modelled sensor locations with error modelling (EEM). The measurement noise was modelled as Gaussian distributed. Standard deviation and mean of the measurement noise was computed from a $N_{t}=263$ time-point section of the recorded signal preceding the illumination. The prior model used in the reconstructions was the Ornstein-Uhlenbeck process. The mean, standard deviation and characteristic length for the prior model were set to $\eta_{p_{0}}=0.04, \sigma_{p_{0}}=0.03$ and $\ell=300 \mu \mathrm{m}$ respectively.

Statistics of the approximation error were simulated using (19)-(20) and $N=10000$ samples of initial pressure $p_{0}$ drawn from the Ornstein-Uhlenbeck prior model. For the forward solutions $K\left(\gamma^{(l)}\right) m^{(l)}$ sensor locations were drawn from a uniform distribution $\mathcal{U}(-188 \mu \mathrm{m}, 188 \mu \mathrm{m})$. The sensor locations were drawn independently for each sensor. To optimise the computational time, the samples corresponding to the altered sensor locations were computed as follows. First three distinct sensor geometries with constant radial displacements of $-188 \mu \mathrm{m}$ 

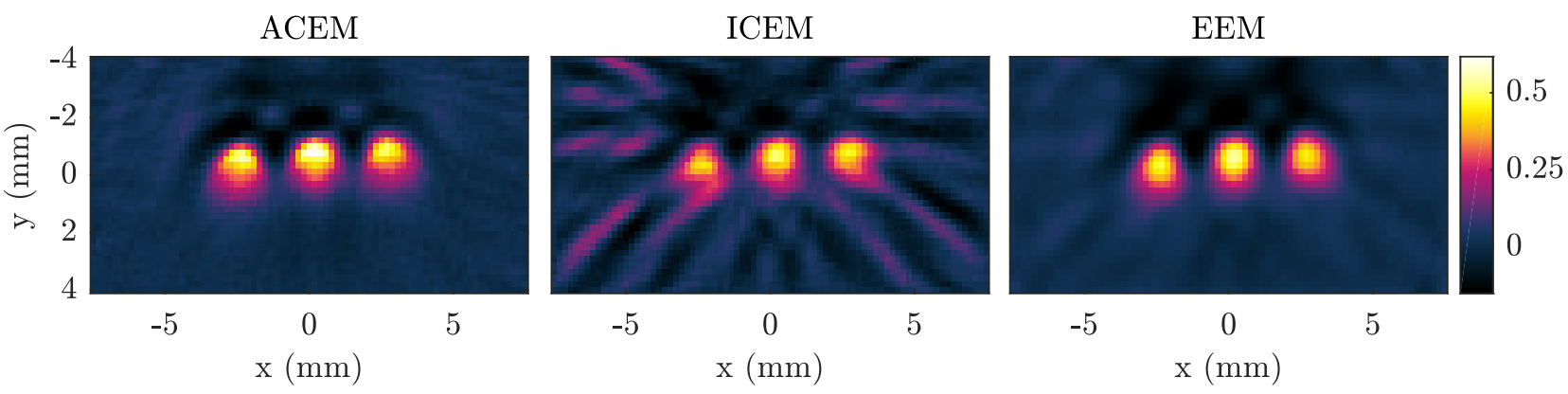

Figure 5: Initial pressure $p_{0}$ estimated from the experimental data. From left to right: the conventional error model with accurately modelled sensor locations (ACEM), the conventional error model with inaccurately modelled sensor locations (ICEM) and the inaccurately modelled sensor locations with error modelling (EEM).

$\left(\mathrm{SG}_{1}\right), 0 \mu \mathrm{m}\left(\mathrm{SG}_{2}\right)$ and $188 \mu \mathrm{m}\left(\mathrm{SG}_{3}\right)$ were constructed. Then $N=10000$ forward solutions using the drawn prior samples were computed for each of the sensor geometries $\mathrm{SG}_{1-3}$. The forward solutions corresponding to $K\left(\gamma^{(l)}\right) m^{(l)}$ were then computed by interpolating the waveforms $\mathrm{SG}_{1-3}$ based on the drawn radial displacement. The forward solutions $K\left(\gamma_{0}\right) m^{(l)}$ were chosen from the forward solutions computed using the sensor geometries $\mathrm{SG}_{1-3}$. These forward solutions were chosen based on the sensor alterations used in the computation of the forward operator $K$.

\subsection{Results}

Means of the posterior distributions computed using experimental data and accurately modelled sensor location (ACEM), inaccurately modelled sensor locations (ICEM) and inaccurately modelled sensor locations with error modelling (EEM) are shown in Fig. 5. As can be seen, the uncertainties in the modelling of ultrasound sensor locations result in wave-like artefacts and distortions of the tube boundaries. These artefacts and distortions are, however, well-compensated using the approximation error modelling. In this case, the shape of the tubes are closer to the true shapes and the wave-like artefacts are corrected. The amplitude of the tubes in the reconstructions using error modelling are, however, somewhat lower and the tube boundaries suffer from slight blurring compared to the reconstructions using accurately modelled sensor locations.

In general, experimental setups can introduce additional possible sources of modelling errors. These sources include factors such as inaccurately measured water temperature, inhomogeneities in the speed of sound within the imaged target and parameters of the ultrasound sensor (such as finite size and frequency response of the sensor). In this work, however, the uncertainties in the speed of sound had a negligible effect compared to the magnitude of the errors due to the uncertainties in ultrasound sensor locations. Furthermore, the parameters of the ultrasound sensors were taken into account by modelling the finite size and the frequency response of the ultrasound sensor.

\section{CONCLUSIONS}

In this work, compensation of errors due to uncertainties in ultrasound sensor locations were studied. Compensation of the errors was carried using the Bayesian approximation error modelling. The method was studied using various levels of uncertainties and sensor geometries as well as different levels of measurement noise. The approach was evaluated by simulations and experiments using accurately modelled sensor locations, inaccurately modelled sensor locations and inaccurately modelled sensor locations with error modelling.

Uncertainties in ultrasound sensor locations were found to cause significant errors in the solution of the inverse problem in PAT. Furthermore, effects of the modelling errors were found to depend on multiple factors, such as the sensor geometry and the magnitude of the noise and modelling errors. The errors caused by uncertainties in ultrasound sensor locations were, however, well compensated using Bayesian approximation error modelling. Based on the results, the proposed approach could be utilised with measurement setups in which the accurate measurement of ultrasound sensor locations is challenging such as hand-held systems. Furthermore, the results 
indicate, that modelling of the errors can play an increasingly important role in situations, where the level of measurement noise is low. That is, increasing the accuracy of the measurement systems and their signal to noise ratio sets a requirement for more accurate forward modelling and modelling of errors.

\section{REFERENCES}

[1] Wang, L. V. and Yao, J., "A practical guide to photoacoustic tomography in the life sciences," Nature Methods 13(8), 627-638 (2016).

[2] Beard, P., "Biomedical photoacoustic imaging," Interface Focus 1(4), 602-631 (2011).

[3] Cox, B. T., Laufer, J. G., Arridge, S. R., and Beard, P. C., "Quantitative spectroscopic photoacoustic imgaging: a review," J. Biomed. Opt. 17(6), 061202 (2012).

[4] Jin, X. and Wang, L. V., "Thermoacoustic tomography with correction for acoustic speed variations," Phys. Med. Biol. 51(24), 6437-6448 (2006).

[5] Matthews, T. P., Poudel, J., Li, L., Wang, L. V., and Anastasio, M. A., "Parametrized joint reconstruction of the initial pressure and sound speed distributions for photoacoustic computed tomography," Soc. Ind. Appl. Math. 11(2), 1560-1588 (2018).

[6] Tick, J., Pulkkinen, A., and Tarvainen, T., "Modelling of errors due to speed of sound variations in photoacoustic tomography using a Bayesian framework," Biomed. Phys. Eng. Express 6(1), 015003 (2019).

[7] Wang, K., Su, R., Oraevsky, A. A., and Anastasio, M. A., "Investigation of iterative image reconstruction in three-dimensional optoacoustic tomography," Phys. Med. Biol. 57(17), 5399-5423 (2012).

[8] Mitsuhashi, K., Wang, K., and Anastasio, M. A., "Investigation of the far-field approximation for modeling a transducer's spatial impulse response in photoacoustic computed tomography," Photoacoustics 2(1), 21-32 (2014).

[9] Kaipio, J. P. and Somersalo, E., [Statistical and Computational Inverse Problems], Springer, New York, NY, USA (2005).

[10] Sahlström, T., Pulkkinen, A., Tick, J., Leskinen, J., and Tarvainen, T., "Modeling of errors due to uncertainties in ultrasound sensor locations in photoacoustic tomography," IEEE T. Med. Imaging, (Accepted for publication, 2020).

[11] Pierce, A. D., [Acoustics - An introduction to physical principles and its applications], Melville, Ny, USA: Acoust. Soc. Am. (1981).

[12] Treeby, B. E. and Cox, B. T., "k-Wave: MATLAB toolbox for the simulation and reconstruction of photoacoustic wave fields," J. Biomed. Opt. 15(2), 021314 (2010).

[13] Rasmussen, C. E. and Williams, C. K. I., [Gaussian Processes for Machine Learning], Cambridge, MA, USA: MIT Press (2006).

[14] Leskinen, J., Pulkkinen, A., Tick, J., and Tarvainen, T., "Photoacoustic tomography setup using LED illumination," in [Opto-Acoustic Methods and Applications in Biophotonics IV], Ntziachristos, V. and Zemp, R., eds., 11077, 57 - 68 (2019). 